\title{
003 REVIEW OF CARDIOVASCULAR DISEASE PRIMARY PREVENTION INTERVENTIONS IN LOW- AND MIDDLE-INCOME COUNTRIES
}

J Park, ${ }^{1}$ M Oremus ${ }^{2} .{ }^{1}$ Honours' Bachelor of Health Sciences Program, Faculty of Health Sciences, McMaster University, Ontario, Canada; ${ }^{2}$ Department of Clinical Epidemiology and Biostatistics, McMaster University, Ontario, Canada

\subsection{6/jech-2014-205217.3}

Background The burden of cardiovascular diseases (CVD) in low- and middle-income countries (LMIC) has increased greatly. Primary prevention of CVD has been shown to work in developed countries, and is urgently needed in LMIC.

Objective This literature review aims to evaluate the effectiveness of primary prevention strategies for CVD in LMIC.

Methods Studies were obtained via a detailed search using Ovid MEDLINEC, EMBASE and Global Health databases, published in English between 1946 and 2013. Included studies were primary research articles aimed at the primary prevention of CVD in LMIC participants over age 15 years. We included studies of interventions targeted to the general population or to groups with specific levels of risk for CVD.

Results Of 1002 studies that were identified through the initial search, 12 studies met the inclusion criteria. The interventions at the general population level mainly consisted of health promotion and education through media, as well as policy implementation and community outreach. The risk-group focused interventions used pharmaceutical treatment of cardiovascular risk factors or CVD education and lifestyle counselling as preventive measures. All but two studies showed significant improvement in at least one cardiovascular risk factor. Primary prevention strategies used in high-risk groups showed greater benefits in reducing physiological cardiovascular risk factors when compared to population-based interventions.

Conclusion Primary prevention interventions have been shown to be effective at lowering cardiovascular risk factors in LMIC. In 
general, the included studies indicate that health promotion and education are the most successful prevention strategies. However, due to heterogeneity between the included studies (e.g. different populations or treatment modalities), no valid conclusions on the comparative effectiveness of specific study interventions can be made. 